\title{
Abscesso extradural da coluna vertebral - Revisão de literatura
}

\author{
Carlos Umberto Pereira ${ }^{1}$ \\ Serviço de Neurocirurgia do HUSE, Aracaju, SE.
}

\section{RESUMO}

O abscesso extradural da coluna vertebral tem sido considerado raro. Sua incidência é de 0,2 a 2 casos por 10.000 internações/ano. O presente trabalho apresenta uma revisão sobre abscesso extradural da coluna vertebral, analisando causas, quadro clínico, exames de neuroimagens, tratamento e prognóstico. Trata-se de artigo de revisão baseado em levantamento nas bases de dados Medline/PubMed e SciELO, em que foram analisados os artigos mais relevantes a respeito desse assunto. Foram selecionados 66 artigos, nos quais são analisados fatores predisponentes, localização anatômica, quadro clínico, exames complementares, tratamento e prognóstico. Acomete mais indivíduos idosos e do sexo masculino. Os principais fatores predisponentes são: diabetes mellitus, pacientes imunodeprimidos e intervenções cirúrgicas na coluna vertebral. As condições clínicas pré-operatórias, intervenção cirúrgica precoce, antibioticoterapia apropriada e reabilitação intensiva são fatores determinantes no prognóstico em abscesso extradural da coluna vertebral.

\section{PALAVRAS-CHAVE}

Abscesso epidural, coluna vertebral/terapia, compressão da medula espinal, imagem por ressonância magnética.

\section{ABSTRACT}

Spinal epidural abscess - Review of literature

Extradural abscess in spine is considered rare. Its incidence is 0.2 to 2 cases in 10.000 hospitalization/ year. The present research presents a review about extradural abscess in spine, analyzing causes, clinical state, neuroimaging exams, treatment and prognosis. It's a review article based in Medline/PubMed and SciELO data, were analyzed the most relevant articles about the present subject. It was analyzed 66 articles, analyzing predisposing factors, anatomical location, clinical state, complementary exams, treatment and diagnosis. Affects mainly the elderly and males. The main predisposing factors are: diabetes mellitus, immunodepressed patients and surgical intervention in spine. Clinical pre-operative conditions, precocious surgical intervention, appropriate antibiotic therapy and intensive rehabilitation are determinant in prognosis of spine extradural abscess.

\section{KEYWORDS}

Epidural Abscess, spine/therapy, spinal cord compression, magnetic resonance imaging.

\section{Considerações gerais}

O abscesso extradural da coluna vertebral (AECV) é uma infecção localizada no espaço extradural. Foi descrito pela primeira vez por Morgagni, no século XIX. Tem sido considerado como a principal causa de AECV o diabetes mellitus, ocorrendo entre $18 \%$ e $54 \%$ dos casos ${ }^{1-7}$ seguido de pacientes imunodeprimidos, uso de drogas ilícitas por via venosa, procedimentos cirúrgicos na coluna vertebral, alcoolismo crônico, insuficiência renal crônica e trauma (Tabela 1). ${ }^{3,8-11} \mathrm{Em} 20 \%$ dos casos não é observado o fator predisponente..$^{6,12-16}$ Sua mortalidade varia de $7 \%$ a $31 \%$ dos casos. . $^{4,6,8,11,17-23}$

Seu diagnóstico é feito por meio do exame clínico e de exames de neuroimagens. O diagnóstico precoce

\begin{tabular}{lc}
\hline \multicolumn{2}{c}{ Tabela 1 - Fatores predisponentes para AEC $\boldsymbol{~}^{*}$} \\
\hline Fatores predisponentes & Frequência (\%) \\
\hline Infecção & $\mathbf{4 4}$ \\
Piodermite & \\
Discite/osteomielite vertebral & \\
Infecção pulmonar/mediastinal & \\
Sepse & 34 \\
Imunodeficiência & \\
Diabetes mellitus & \\
Uso de droga intravenosa & \\
Alcoolismo crônico & \\
Insuficiência renal crônica & \\
AIDS & $\mathbf{2 2}$ \\
Procedimento invasivo de coluna/cirurgia & $\mathbf{1 0}$ \\
Trauma & $\mathbf{2 0}$ \\
Ausência de fatores predisponentes &
\end{tabular}

* Fonte: Reihaus E, Waldbaur H, Seeling W. Spinal epidural abscess: a meta-analysis of 915 patients. Neurosurg Rev. 2000;232:175-204.

1. Professor doutor adjunto do Departamento de Medicina da Universidade Federal de Sergipe (UFS), neurocirurgião do Serviço de Neurocirurgia do Hospital João Alves Filho, Aracaju, SE. 
seguido de um tratamento eficiente tem sido importante para que se obtenha um excelente resultado.

\section{Epidemiologia}

O AECV está constituído por uma coleção purulenta situada no espaço extradural e pode estar localizado em um ou vários segmentos da coluna vertebral ou estender-se para toda coluna vertebral. É raro e ocorre entre 0,2 e 2 casos por 10.000 admissões hospitalares/ ano. ${ }^{2,3,6-8,10,15,17,24-31}$ Cerca de $5 \%$ a $18 \%$ dos casos de osteomielite vertebral ou de discite podem desenvolver por contiguidade um AECV. Atualmente, tem sido demonstrado um aumento em sua incidência devido ao número de intervenções cirúrgicas por instrumentação e ao aumento do uso abusivo de drogas por via endovenosa. ${ }^{8,10,11,13,28,32-34}$ Também tem sido denominado de peripaquimeningite espinhal, paquimeningite espinhal externa e perimeningite espinhal. ${ }^{6}$

O AECV acomete qualquer faixa etária, porém apresenta predileção por indivíduos com idades acima de 50 anos. ${ }^{2,6,10,11,13,33}$ Acomete mais o gênero masculino, ${ }^{6,10,19,33}$ provavelmente devido a uma maior incidência de fatores predisponentes nesse gênero como alcoolismo crônico, uso de drogas ilícitas e maior predisposição aos traumatismos. ${ }^{2}$ Fatores cirúrgicos de riscos no desenvolvimento de AECV incluem: operação de longa duração, grande perda sanguínea, uso de instrumentação ou múltiplas revisões ou atos cirúrgicos. ${ }^{10,30,35,36}$

\section{Localização}

A localização mais comum do AECV é a região dorsal (50\%-80\%), provavelmente devido à maior extensão anatômica e pelo maior desenvolvimento do plexo venoso nessa localização, seguido da região lombossacra (17\%-38\%) e cervical (15\%) (Figura 1). ${ }^{6,10,13,16,30} \mathrm{Na}$ região cervical, geralmente se encontra associado com a presença de osteomielite vertebral. O AECV localizado anteriormente se encontra geralmente associado à osteomielite vertebral e discite, em $80 \%$ dos casos. ${ }^{2} \mathrm{O}$ AECV localiza-se normalmente na parte posterior do saco dural (80\%), sendo menos comum na sua porção anterior (20\%). ${ }^{6,10,13,33}$ Os AECVs localizados na porção posterior se estendem axialmente com mais facilidade que os de localização anterior, devido à estreita união da dura-máter com a parte óssea da coluna e dos ligamentos da porção espinhal anterior. ${ }^{37}$ Pode acometer apenas um corpo vertebral ou afetar toda extensão da coluna vertebral. ${ }^{12}$

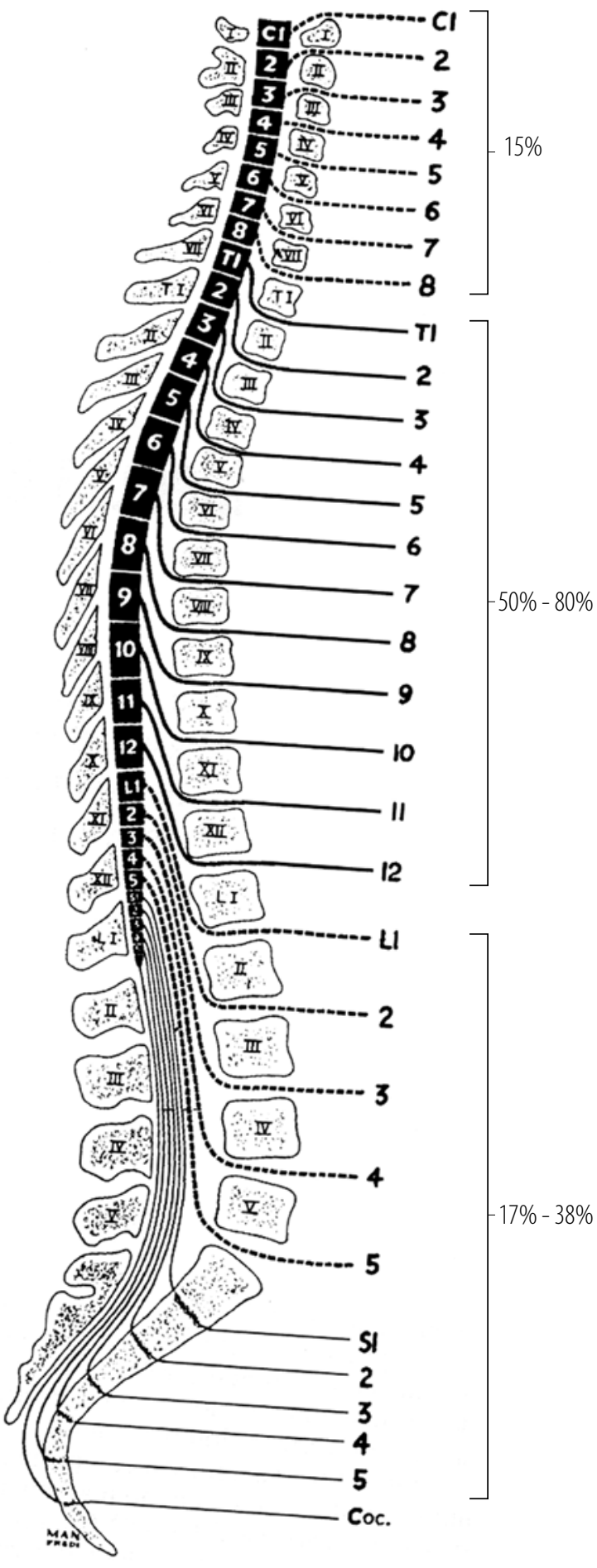

Figura 1 - Localização.

\section{Vias de disseminação}

Os microrganismos podem alcançar o espaço extradural da coluna vertebral de três maneiras: 1 ) via hematogênica: a partir de focos infecciosos a distância, como infecção de partes moles, respiratórias, urinárias 
e otorrinolaringológicas. É a via de disseminação mais comum; 2) por contiguidade: por meio da extensão direta ou pelo plexo venoso epidural de infecções próximas ao canal vertebral, como as infecções paravertebrais, do músculo psoas e os retrofaríngeos ou por extensão local da osteomielite vertebral adjacente; e 3) por inoculação direta: situações em que ocorre laceração do espaço extradural por traumatismos, procedimentos cirúrgicos, punção lombar, discografia e anestesia epidural. ${ }^{6-8,10,26,30,38}$

\section{Microrganismos}

O AECV pode ser devido a fungos, bactérias e vírus (Quadro 1). O Staphylococcus aureus tem sido o microrganismo mais comumente envolvido (60\% a $70 \%$ dos casos) $3,7,10,13-17,19,24,28,31,38,39$, seguido por diferentes tipos de Streptococcus e microrganismos Gram-negativos como Escherichia coli, Mycobacterium tuberculosis, fungos e raramente helmintos podem causar $\mathrm{AECV}^{6-8,8,19,36}$

Quadro 1-Microrganismos causadores de AECV

\begin{tabular}{l} 
Bactérias \\
\hline Staphylococcus aureus \\
Coagulase-negative staphylococci \\
Bacilos entéricos Gram-negativos \\
Pseudomonas aeruginosa \\
Streptococcus pneumoniae \\
Streptococcus pyogenes \\
Viridans e outros Streptococci \\
Listeria monocytogenes \\
Salmonella species \\
Brucella suis \\
Actinomyces \\
Anaerobic bacteria \\
Mycobacteria \\
Fungos \\
Aspergillus species \\
Blastomyces dermatitides \\
Coccidioides immitis \\
Cryptococcus neoformans \\
Mucormycosis \\
Eelmintos \\
Drinococcus granulosus \\
\hline
\end{tabular}

\section{Sintomatologia}

Sua apresentação clínica pode ser inespecífica, retardando, assim, seu diagnóstico e favorecendo um péssimo prognóstico. Geralmente, manifesta-se por dor localizada, febre e sensibilidade local. ${ }^{6,8,12,13,40} \mathrm{~A}$ dor pode ser radicular, no trajeto da raiz nervosa acometida pela infecção. Pode desenvolver paresia ou paralisia muscular e alterações esfincterianas.

Pode se apresentar clinicamente de forma aguda ou crônica. ${ }^{41} \mathrm{Na}$ forma aguda, tem evolução clínica grave de poucos dias, manifestando-se geralmente com quadro de dor nas costas do tipo radicular e febre, podendo desenvolver quadro agudo de paresia, transtornos sensitivos e alterações esfincterianas; ${ }^{38,42}$ nessa fase a dor pode estar ausente entre $10 \%$ e $15 \%$ dos $\operatorname{casos}^{11,38}$, e em $50 \%$ dos casos ausência de febre pode ocorrer; ${ }^{6,11,17,38}$ ocasionalmente, nessa fase pode confundir seu diagnóstico com um quadro de sepse, retardando, assim, seu diagnóstico e tratamento adequado, ${ }^{10,37}$ e na evolução crônica desenvolve sintomas no decorrer de várias semanas, bem como síndrome de compressão medular de evolução lenta.

\section{Exames laboratoriais}

O hemograma demonstra leucocitose com ligeira neutrofilia e velocidade de hemossedimentação (VHS) elevada, ${ }^{6,710,17,33,38,43}$ assim como a proteína C-reativa. ${ }^{4,530}$; identificação do microrganismo na cultura do sangue ou mesmo do local da infecção, com amostra colhida por meio de biópsia guiada por tomografia computadorizada (TC). ${ }^{13}$ A cultura do sangue é positiva em $82 \%$ dos casos. ${ }^{44}$

\section{Exames de neuroimagem}

Exames de imagens obtidas mediante ressonância magnética (RM) com reforço endovenoso de gadolínio têm sido considerados os exames de eleição para diagnóstico e conduta. ${ }^{1,6,7,10,14,17,25-28,38,45-49}$ Apresenta sensibilidade diagnóstica de $91 \%{ }^{3,6}$ A RM com meio de contraste é superior à TC, porque delineia o $\mathrm{AECV}$ das estruturas contíguas e diferencia o AECV de tumor espinhal, hematomas, mielite transversa, infarto medular e prolapso de disco intervertebral. ${ }^{6,13}$ A RM demonstra uma lesão extradural iso ou hipointensa em T1 e hiperintensa em T2, produzindo efeito de massa ${ }^{17,45,50}$ (Figuras 2 e 3). A porção líquida do abscesso é geralmente hiperintensa em T2 com correspondente hipointenso em T1..$^{27} \mathrm{O}$ uso de contraste é de suma importância para definir a idade e consistência do abscesso. A parte líquida do abscesso está associada com uma área de baixa intensidade em $\mathrm{T} 1$, enquanto a cápsula do abscesso que reforça após a injeção de gadolínio representa tecido de granulação. ${ }^{27}$ 


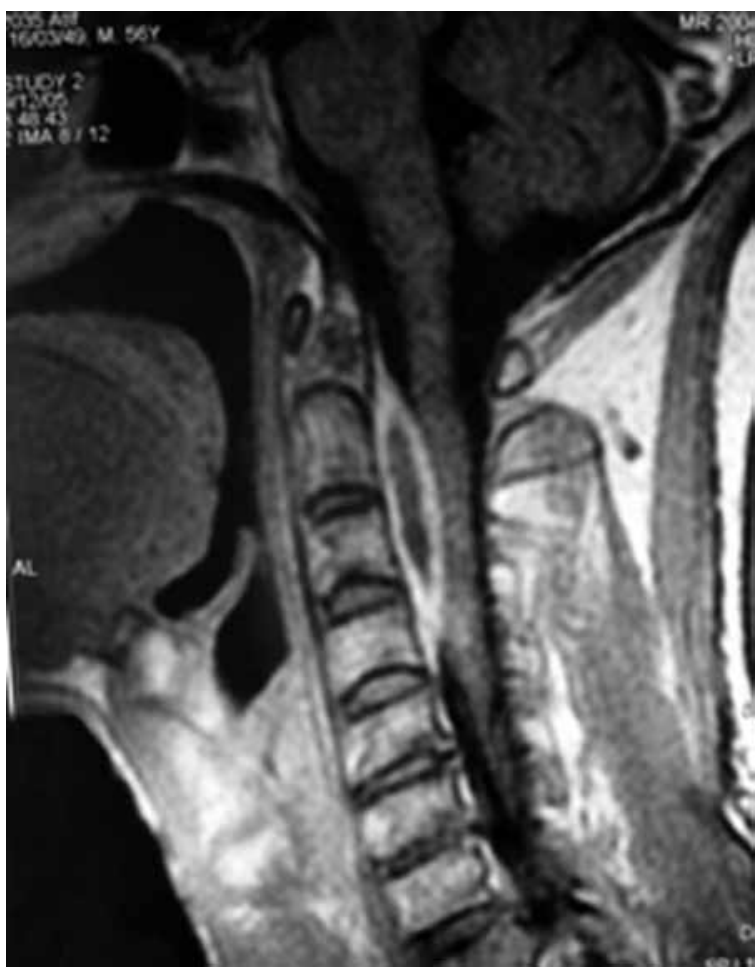

Figura 2 - Ressonância magnética da coluna vertebral em T1 demonstrando abscesso epidural cervical.

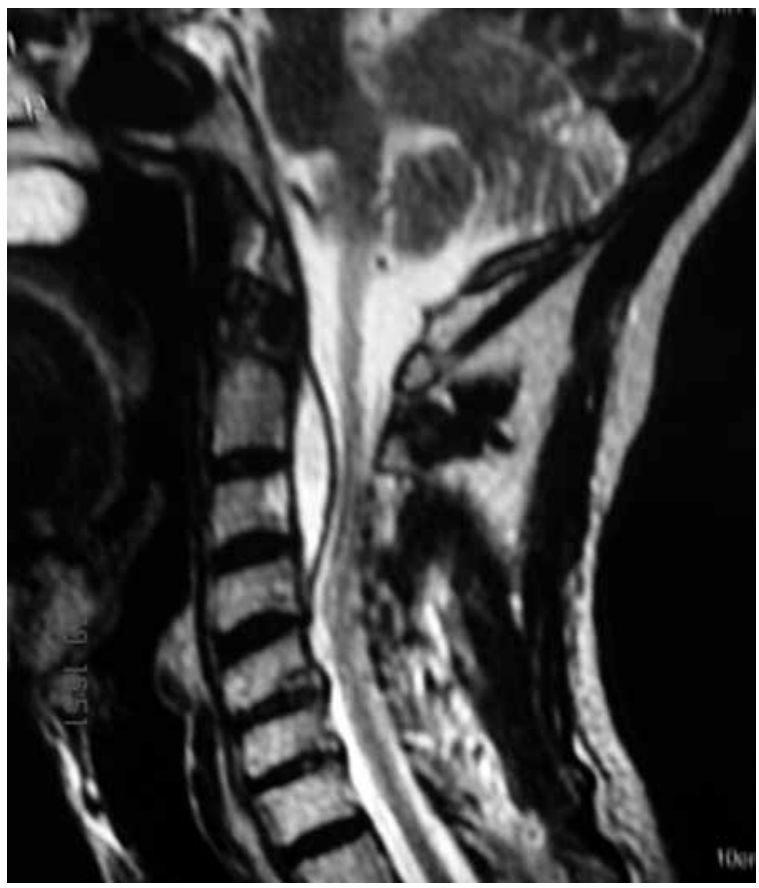

Figura 3 - Ressonância magnética da coluna cervical em T2 apresentando abscesso epidural cervical.

O AECV associa-se geralmente com uma compressão da medula espinhal ao exame de RM. ${ }^{25}$ Mielotomografia tem sido utilizada, porém apresenta risco elevado de introduzir microrganismo dentro do espaço intratecal na presença de AECV localizado na região lombar. ${ }^{13,38}$

\section{Diagnóstico diferencial}

O AECV apresenta diagnóstico diferencial com abscesso ou neoplasia intramedular, paquimeningite hipertrófica espinhal, meningite, empiema subdural, hematoma epidural, mielite transversa e osteomielite vertebral. ${ }^{36,41}$

\section{Tratamento}

O tratamento do AECV inclui imobilização, descompressão cirúrgica com ou sem drenagem do abs-

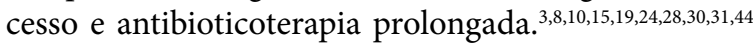
Ocasionalmente, estabilização da coluna vertebral é necessária. ${ }^{3,8,15,24,30,31} \mathrm{O}$ início do tratamento é geralmente tardio devido à dificuldade de reconhecer precocemente essa doença. ${ }^{8,13,30,51}$ Imobilização é recomendada se existe dor significante ou potencial risco de instabilidade da coluna. O tratamento clínico está indicado em caso de ausência de sinais neurológicos deficitários, quando o paciente tem pouco ou nenhum déficit neurológico focal, e segundo, quando a taxa de morbidade e mortalidade da indicação de intervenção cirúrgica é elevada. ${ }^{8,14,52,53}$

Estabelecida a indicação de tratamento clínico, usam-se antibióticos ou por via endovenosa pelo período mínimo de seis a oito semanas, dependendo, sobretudo, da resposta clínica, sendo necessários controle seriado de VHS e proteína C-reativa. ${ }^{33,35,52,54}$ Em casos de osteomielite vertebral associada, estende-se o tratamento por mais dez semanas. ${ }^{10,17,26,33}$ Leys et al. ${ }^{55}$ propuseram tratamento clínico em casos em que não exista fator de risco importante de contraindicação cirúrgica, casos em que a extensão da lesão seja toda coluna vertebral, que o déficit neurológico seja completo e com mais de três dias de evolução ou que não exista um déficit neurológico significativo. Tem sido usado antibiótico por via sistêmica por meio da combinação de cefalosporina de terceira geração associada a um agente antiestafilocócico, e, eventualmente, metronidazol, pois permitem cobrir os microrganismos que frequentemente desenvolvem o AECV. Um regime empírico apropriado, inicialmente, inclui cloxacilina com metronidazol e cefotaxime ou ceftazidime. ${ }^{6,30}$ Vancomicina pode ser substituída por cloxacilina em casos de alergia a penicilina ou se o $S$. aureus for meticilina-resistente. ${ }^{6,30}$ Siddiq et al. ${ }^{56}$ recomendam tratamento conservador nas seguintes situações: ausência de déficit neurológico, paralisia completa com mais de 72 horas de evolução, identificação do microrganismo responsável por meio de outras fontes, lesão extensa no canal medular, pacientes com alto risco cirúrgico. Porém, em casos 
de surgimento de sintomas neurológicos progressivos, a descompressão cirúrgica está indicada de imediato. ${ }^{13}$

A maioria dos autores indica tratamento cirúrgico imediato, associado à antibioticoterapia sistêmica por seis a oito semanas, antes do desenvolvimento de déficits neurológicos. ${ }^{19,36,41} \mathrm{O}$ tratamento cirúrgico encontra-se indicado quando: 1) existe envolvimento ósseo significante, 2) déficit neurológico, 3) sepse com sinais clínicos de toxicidade causada por abscesso que não responde à terapia medicamentosa, 4) dificuldade para obter material purulento para cultura por meio de punção com agulha e 5) insuficiência de o antibiótico só combater à infecção. $\mathrm{O}$ tratamento cirúrgico tradicional envolve laminectomia com ou sem estabilização da coluna. Quando os elementos da porção anterior da coluna estão envolvidos pelo processo infeccioso, destruição óssea e de disco intervertebral, podem ser necessárias realização de corpectomia e estabilização da coluna. ${ }^{3,15,31}$ Deve-se evitar, durante $\mathrm{o}$ ato cirúrgico, a abertura da dura-máter, para evitar a disseminação do processo infeccioso para o interior do saco dural. ${ }^{6,33,43,56}$ Técnicas minimamente invasivas como laparoscopia e toracoscopia têm sido hoje utilizadas, assim como procedimentos vídeo-assistidos. ${ }^{57-59}$ A ultrassonografia intraoperatória tem sido utilizada com sucesso. ${ }^{48,60} \mathrm{~A}$ inserção de cateter no espaço epidural e no abscesso pode ser feita por técnicas a céu aberto ou por meio de técnicas percutâneas. ${ }^{61-63}$ Portanto, o procedimento cirúrgico está orientado para drenagem do abscesso, que, assim, alivia a compressão medular e obtém material para identificação bacteriológica do microrganismo causador do AECV. ${ }^{6,33,38}$

Os objetivos do procedimento cirúrgico são: realizar desbridamento do tecido infectado (limpeza e remoção), tornar a área acometida apta para receber fluxo sanguíneo adequado, para restabelecer sua cicatrização, manter e restaurar, se necessário, sua estabilidade e limitar o grau de deterioração neurológica. ${ }^{6,33}$

\section{Prognóstico}

São considerados fatores de prognósticos no AECV: 1) quadro clínico: o prognóstico é melhor em pacientes que apresentam quadro doloroso e pior em casos de paralisias, ${ }^{33}$ 2) grau de compressão do saco dural: pior prognóstico em caso de ocupação em mais de $50 \%$ do diâmetro do canal, ${ }^{8,33,64}$ 3) duração dos sintomas antes do tratamento: o início do tratamento antes de 72 horas do começo dos sintomas relaciona-se com melhor prognóstico, e a compressão medular com mais de 72 horas pode induzir formação de trombose venosa e infarto medular, tornando o déficit neurológico irreversível, ${ }^{33,65} 4$ ) idade do paciente: pior prognóstico em pacientes acima de 50 anos - os jovens possuem maior plasticidade medular e, assim, toleram melhor a compressão medular, ${ }^{18}$ 5) localização: os AECVs localizados na região cervical possuem pior prognóstico que os dorsal e lombar, 6) tratamento: conforme dados da literatura, os pacientes submetidos a tratamento clínico apresentam melhores resultados que os tratados cirurgicamente. ${ }^{8}$

Tung et al. ${ }^{64}$ estudaram os achados de RM em pacientes portadores de AE e correlacionaram com péssimo prognóstico os casos em que há presença de captação de contraste no espaço extradural, redução de mais de $50 \%$ do calibre do canal vertebral e uma extensão craniocaudal maior de $3 \mathrm{~cm}$. Complicações cirúrgicas como cifose pós-laminectomia têm sido relatadas em algumas séries. ${ }^{3,31,51,58,62,66}$ Seu prognóstico também depende fundamentalmente da demora em iniciar o tratamento, do estado clínico do paciente por ocasião do diagnóstico, da idade e da associação com patologias sistêmicas. ${ }^{2}$ Khanna et al. ${ }^{8}$ estabeleceram uma escala com valor prognóstico baseados na idade, grau de compressão do saco dural e tempo de evolução dos sintomas (Tabela 2). O diagnóstico tardio ocorre em $68 \%$ dos pacientes, o qual contribui para $45 \%$ de morbidade e $15 \%$ de mortalidade. ${ }^{6,11}$

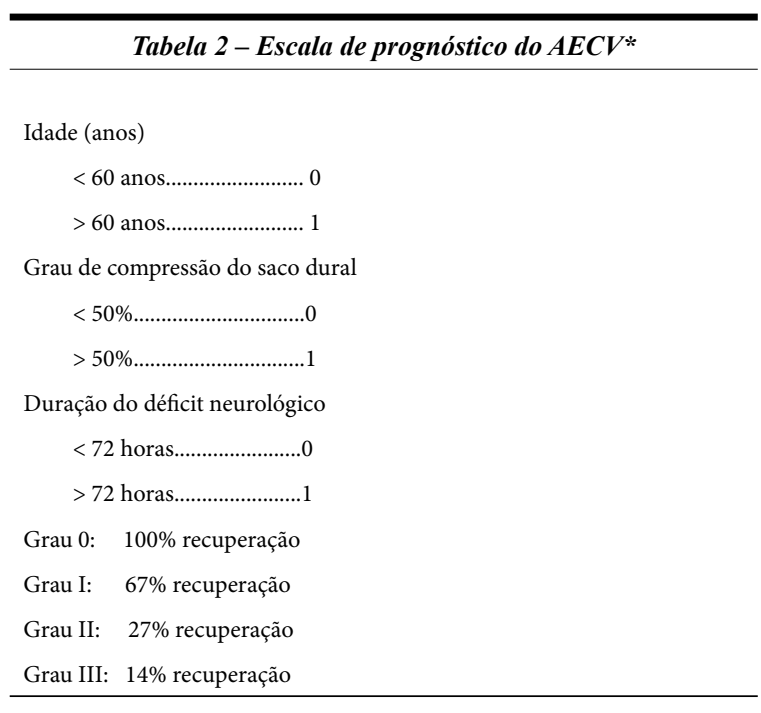

* Fonte: Khanna RK, Malik GM, Rock JP, Rosenblum ML. Spinal epidural abscess: evaluation of factors influencing outcome. Neurosurgery. 1996;39:958-64.

\section{Considerações finais}

Como o tratamento do AECV pode ser clínico ou cirúrgico, é necessária uma equipe multidisciplinar que inclua infectologistas, neurorradiologistas e neurocirurgiões especializados em cirurgia vertebral para que ocorra sucesso nos resultados de pacientes acometidos por AECV. 


\section{Referências}

1. Danner RL, Hartman BJ. Update on spinal epidural abscess: 35 cases and review of the literature. Rev Infect Dis. 1987;9(2):265-74.

2. Gelabert-González M, González-García J, Fernández-Villa JM, GarcíaAallut A, Martínez-Rumbo R. [Spinal epidural empyema. Analysis of 14 cases]. Neurocirugia (Astur). 2004;15(6):543-51.

3. Hlavin ML, Kaminski HJ, Ross JS, Ganz E. Spinal epidural abscess: a ten-year perspective. Neurosurgery. 1990;27(2):177-84.

4. Joshi SM, Hatfield RH, Martin J, Taylor W. Spinal epidural abscess: a diagnostic challenge. $\mathrm{Br} \mathrm{J}$ Neurosurg. 2003;17(2):160-3.

5. Pilkington SA, Jackson SA, Gillett GR. Spinal epidural empyema. Br J Neurosurg. 2003;17(2):196-200.

6. Reihsaus E, Waldbaur H, Seeling W. Spinal epidural abscess: a meta-analysis of 915 patients. Neurosurg Rev. 2000;23(4):175-204.

7. Tang HJ, Lin HJ, Liu YC, Li CM. Spinal epidural abscess experience with 46 patients and evaluation of prognostic factors. J Infect. 2002;45(2):76-81.

8. Khanna RK, Malik GM, Rock JP, Rosenblum ML. Spinal epidural abscess: evaluation of factors influencing outcome. Neurosurgery. 1996;39(5):958-64.

9. Parkinson JF, Sekhon LH. Spinal epidural abscess: appearance on magnetic resonance imaging as a guide to surgical management. Report of five cases. Neurosurg Focus. 2004;17(6):E12.

10. Rigamonti D, Liem L, Sampath P, Knoller N, Namaguchi Y, Schreibman DL, et al. Spinal epidural abscess: contemporary trends in etiology, evaluation, and management. Surg Neurol. 1999;52(2):189-96.

11. Soehle M, Wallenfang T. Spinal epidural abscesses: clinical manifestations, prognostic factors, and outcomes. Neurosurgery. 2002;51(1):79-85.

12. Akalan N, Ozgen T. Infection as a cause of spinal cord compression: a review of 36 spinal epidural abscess cases. Acta Neurochir (Wien). 2000;142(1):17-23.

13. Chao D, Nanda A. Spinal epidural abscess: a diagnostic challenge. Am Fam Physician. 2002;65(7):1341-6.

14. Mackenzie AR, Laing RB, Smith CC, Kaar GF, Smith FW. Spinal epidural abscess: the importance of early diagnosis and treatment. J Neurol Neurosurg Psychiatry. 1998;65(2):209-12.

15. Martin RJ, Yuan HA. Neurosurgical care of spinal epidural, subdural, and intramedullary abscesses and arachnoiditis. Orthop Clin North Am. 1996;27(1):125-36.

16. Vilke GM, Honingford EA. Cervical spine epidural abscess in a patient with no predisposing risk factors. Ann Emerg Med. 1996;27(6):777-80.

17. Darouiche RO, Hamill RJ, Greenberg SB, Weathers SW, Musher DM. Bacterial spinal epidural abscess. Review of 43 cases and literature survey. Medicine (Baltimore). 1992;71(6):369-85.

18. Lange M, Tiecks F, Schielke E, Yousry T, Haberl R, Oeckler R. Diagnosis and results of different treatment regimens in patients with spinal abscesses. Acta Neurochir (Wien). 1993;125(1-4):105-14.

19. Pereira CE, Lynch JC. Spinal epidural abscess: an analysis of 24 cases. Surg Neurol. 2005;63(Suppl 1):S26-9.

20. Rothman SL. The diagnosis of infections of the spine by modern imaging techniques. Orthop Clin North Am. 1996;27(1):15-31.
21. Schultz KD Jr, Comey CH, Haid RW Jr. Technical note. Pyogenic spinal epidural abscess: a minimally invasive technique for multisegmental decompression. J Spinal Disord. 2001;14(6):546-9.

22. Schmutzhard E, Aichner F, Dierckx RA, Gerstenbrand F, Willeit J. New perspectives in acute spinal epidural abscess. Illustrated by two case reports. Acta Neurochir (Wien). 1986;80(3-4):105-8.

23. Tacconi L, Johnston FG, Symon L. Spinal epidural abscess - review of 10 cases. Acta Neurochir (Wien). 1996;138(5):520-3.

24. Del Curling O Jr, Gower DJ, McWhorter JM. Changing concepts in spinal epidural abscess: a report of 29 cases. Neurosurgery. 1990;27(2):185-92.

25. Friedmand DP, Hills JR. Cervical epidural spinal infection: MR imaging characteristics. AJR Am J Roentgenol. 1994;163(3):699-704.

26. Barbadillo Izquierdo F, Merino Arribas J, Carpintero Martín I, Rodrigo, Palacios J, González De La Rosa JB. [Successful medical treatment of spinal epidural abscess]. An Esp Pediatr. 2001;54(2):185-7.

27. Lang IM, Hughes DG, Jenkins JP, St Clair Forbes W, McKenna F. MR imaging appearances of cervical epidural abscess. Clin Radiol. 1995;50(7):466-71.

28. Lindner A, Warmuth-Metz M, Becker G, Toyka VV. latrogenic spinal epidural abscesses: early diagnosis essential for good outcome. Eur J Med Res. 1997;2(5):201-5.

29. Messer HD, Lenchner GS, Brust JC, Resor S. Lumbar spinal abscess managed conservatively. Case report. J Neurosurg. 1977;46(6):825-9.

30. Nussbaum ES, Rigamonti D, Standiford H, Numaguchi $Y$, Wolf AL, Robinson WL. Spinal epidural abscess: a report of 40 cases and review. Surg Neurol. 1992;38(3):225-31.

31. Rea GL, McGregor JM, Miller CA, Miner ME. Surgical treatment of the spontaneous spinal epidural abscess. Surg Neurol. 1992;37(4):274-9.

32. Davis DP, Wold RM, Patel RJ, Tran AJ, Tokhi RN, Chan TC, et al. The clinical presentation and impact of diagnostic delays on emergency department patients with spinal epidural abscess. J Emerg Med. 2004;26(3):285-91.

33. González-García J, Gelabert M, Bandín J, Villa JM, Pravos AG. [Cervical epidural abscess as a cause of tetraparesis]. Rev Neurol. 1999;29(8):727-30.

34. Koppel BS, Tuchman AJ, Mangiardi JR, Daras M, Weitzner I. Epidural spinal infection in intravenous drug abusers. Arch Neurol. 1988;45(12):1331-7.

35. Louis A, Fernandes CM. Spinal epidural abscess. CJEM. 2005;7(5):351-4.

36. Verner EF, Musher DM. Spinal epidural abscess. Med Clin North Am. 1985;69(2):375-84.

37. Figueroa L, Lorenzo M, Martinez D, Alberione F. El absceso epidural espinal: nuestra experiencia en un año. Rev Argent Neuroc. 2006;20(1):37-40.

38. Curry WT Jr, Hoh BL, Amin-Hanjani S, Eskandar EN. Spinal epidural abscess: clinical presentation, management, and outcome. Surg Neurol. 2005;63(4):364-71.

39. Sampath P, Rigamonti D. Spinal epidural abscess: a review of epidemiology, diagnosis, and treatment. J Spinal Disord. 1999;12(2):89-93.

40. Lu CH, Chang WN, Lui CC, Lee PY, Chang HW. Adult spinal epidural abscess: clinical features and prognostic factors. Clin Neurol Neurosurg. 2002;104(4):306-10.

41. Baker AS, Ojemann RG, Swartz MN, Richardson EP Jr. Spinal epidural abscess. N Engl J Med. 1975;293(10):463-8.

42. Urrutia J, Rojas $\mathrm{C}$. Extensive epidural abscess with surgical treatment and long term follow up. Spine J. 2007;7(6):708-11 . 
43. Löhr M, Reithmeier T, Ernestus RI, Ebel H, Klug N. Spinal epidural abscess: prognostic factors and comparison of different surgical treatment strategies. Acta Neurochir (Wien). 2005;147(2):159-66.

44. Sapico FL. Microbiology and antimicrobial therapy of spinal infections. Orthop Clin North Am. 1996;27(1):9-13.

45. Erntell M, Holtås S, Norlin K, Dahlquist E, Nilsson-Ehle I. Magnetic resonance imaging in the diagnosis of spinal epidural abscess. Scand J Infect Dis. 1988;20(3):323-7.

46. Hanigan WC, Asner NG, Elwood PW. Magnetic resonance imaging and the nonoperative treatment of spinal epidural abscess. Surg Neurol. 1990;34(6):408-13.

47. Levi AD, Dickman CA, Sonntag VK. Management of postoperative infections after spinal instrumentation. J Neurosurg. 1997;86(6):975-80.

48. Post MJ, Quencer RM, Montalvo BM, Katz BH, Eismont FJ, Green BA. Spinal infection: evaluation with MR imaging and intraoperative US. Radiology. 1988;169(3):765-71.

49. Redekop GJ, Del Maestro RF. Diagnosis and management of spinal epidural abscess. Can J Neurol Sci. 1992;19(2):180-7.

50. Parkinson JF, Sekhon LH. Surgical management of spinal epidural abscess: selection of approach based on MRI appearance. J Clin Neurosci. 2004;11(2):130-3.

51. McGee-Collett M, Johnston IH. Spinal epidural abscess: presentation and treatment. A report of 21 cases. Med J Aust. 1991;155(1):14-7.

52. Mampalam TJ, Rosegay $\mathrm{H}$, Andrews BT, Rosenblum ML, Pitts LH. Nonoperative treatment of spinal epidural infections. J Neurosurg. 1989;71(2):208-10.

53. Wheeler D, Keiser P, Rigamonti D, Keay S. Medical management of spinal epidural abscesses: case report and review. Clin Infect Dis. 1992;15(1):22-7.

54. Sørensen P. Spinal epidural abscesses: conservative treatment for selected subgroups of patients. $\mathrm{Br} J$ Neurosurg. 2003;17(6):513-8.

55. Leys D, Lesoin F, Viaud C, Pasquier F, Rousseaux M, Jomin $M$, et al. Decreased morbidity from acute bacterial spinal epidural abscesses using computed tomography and nonsurgical treatment in selected patients. Ann Neurol. 1985;17(4):350-5.

56. Siddiq F, Chowfin A, Tight R, Sahmoun AE, Smego RA Jr. Medical vs. surgical management of spinal epidural abscess. Arch Intern Med. 2004;164(22):2409-12.
57. Manfredi PL, Herskovitz S, Folli F, Pigazzi A, Swerdlow ML. Spinal epidural abscess: treatment options. Eur Neurol. 1998;40(1):58-60.

58. Parker LM, McAfee PC, Fedder IL, Weis JC, Geis WP. Minimally invasive surgical techniques to treat spine infections. Orthop Clin North Am. 1996;27(1):183-99.

59. Roselli R, lacoangeli M, Pompucci A, Trignani R, Restuccia D, Di Lazzaro V, et al. Anterior cervical epidural abscess treated by endoscopy-assisted minimally invasive microsurgery via posterior approach. Minim Invasive Neurosurg. 1998;41(3):161-5.

60. Mak KH, Au KK, Fung KY, Chan YW. Spinal epidural abscess: a report of nine cases and the use of intra-operative ultrasonography. Aust N Z J Surg. 1996;66(5):287-90.

61. Hori K, Kano T, Fukushige T, Sano T. Successful treatment of epidural abscess with a percutaneously introduced 4-French catheter for drainage. Anesth Analg. 1997;84(6):1384-6.

62. Leonard J, Kaufman B. Treatment of a holocord epidural abscess. Case illustration. J Neurosurg. 2001;94(1 Suppl): 179 .

63. Walter RS, King JC Jr, Manley J, Rigamonti D. Spinal epidural abscess in infancy: successful percutaneous drainage in a nine-month-old and review of the literature. Pediatr Infect Dis J. 1991;10(11):860-4.

64. Tung GA, Yim JW, Mermel LA, Philip L, Rogg JM. Spinal epidural abscess: correlation between MRI findings and outcome. Neuroradiology. 1999;41(12):904-9.

65. Anand S, Maini L, Agarwal A, Singh T, Dhal AK, Dhaon BK. Spinal epidural abscess - a report of six cases. Int Orthop. 1999;23(3):175-7.

66. Fischer EG, Greene CS Jr, Winston KR. Spinal epidural abscess in children. Neurosurgery. 1981;9(3):257-60.

\section{Endereço para correspondência}

Carlos Umberto Pereira

Av. Augusto Maynard, 245/404, São José

49015-380 - Aracaju, SE

Telefone: (79) 3213-0518

E-mail: umberto@infonet.com.br 